\title{
Alzheimer's disease: addressing a twenty-first century plague
}

\author{
Christopher M. Dobson ${ }^{1}$
}

Received: 11 July 2015/Accepted: 13 July 2015/Published online: 19 August 2015

(C) The Author(s) 2015. This article is published with open access at Springerlink.com

\begin{abstract}
Alzheimer's disease represents one of the greatest challenges to the social fabric and health care systems of the world since the great plagues of the Middle Ages. At the present time, there are some 40 million sufferers from this disease worldwide, but it is predicted that this number will rise to nearly 150 million by 2050 . The predominant reason for this rapidly increasing prevalence is the increase in longevity that has resulted from the tremendous advances in public health and hygiene and in medical and surgical interventions over the last century. This article discusses recent progress in our understanding of the molecular origins of Alzheimer's disease and emerging ideas for new and rational therapeutic strategies by which to combat its onset and progression.
\end{abstract}

Keywords Protein misfolding - Amyloid formation . Neurodegenerative diseases · Protein homeostasis . Aggregation mechanisms

\section{Introduction}

Plagues, or the rapid emergence of outbreaks of fatal diseases, have been a prominent feature of the human condition throughout history (Dobson 2015). The 'biblical'

The 2014 International Feltrinelli Prize for Medicine was awarded by the Accademia Nazionale dei Lincei to Prof. Christopher M. Dobson. This paper is an account of the lecture that the awardee delivered during the presentation ceremony on the 14 of November 2014.

Christopher M. Dobson

cmd44@cam.ac.uk

1 Department of Chemistry, University of Cambridge, Lensfield Road, Cambridge CB2 1EW, UK plagues of Egypt, recorded in the Book of Exodus, are thought to have occurred between ca. 1500 and $1200 \mathrm{BCE}$, although there has been much speculation about the origins of these events, including whether or not they are mythical, or if they could have represented the consequences of a period of climate change. In more recent history, devastating 'plagues' are better documented, such as the 'Plague of Athens' in 430-426 BCE, which followed severe overcrowding in the city, accompanied by insanitary conditions, as people took refuge from the approaching Spartan army during the Peloponnesian War. It is thought that a third of the population of Athens could have died from disease at this time, speculated to be anything from typhoid to measles.

Another well-documented plague from antiquity was the 'Plague of Justinian', which occurred in 541-544 AD in Constantinople and throughout the Byzantine Empire. There is increasing evidence that this pandemic was the first recorded outbreak of bubonic plague, the disease that more than any other is often simply described as 'the plague'. Frequent outbreaks occurred in mediaeval Europe, the infection having spread from Asia along the increasingly heavily used trade routes. The most famous of these episodes was the 'Great Mortality' or the 'Black Death' that afflicted Europe in 1347-1353 AD, and is estimated to have killed one third of the population of the continent and to have caused unprecedented social and economic upheaval. There were other periodic outbreaks of bubonic plague, including the 'Great Plague' of London in 1665, although none was as devastating as that of the mid-fourteenth century. Other diseases, however, ravaged the world, including measles and smallpox, which spread like wildfire amongst the populations of 'new worlds' such as the Americas and Australasia with the arrival of European colonists, as the indigenous people had no immunity to 
these diseases that were previously unknown in those regions (Dobson 2015; Haines and Steckel 2014).

With the massive overcrowding that occurred in cities as a result of the increasing industrialisation in the nineteenth century, outbreaks of infectious diseases such as cholera and typhoid became common until the importance of public health and hygiene became recognised. Then, in the aftermath of the First World War, perhaps the most lethal pandemic of all time occurred, the 'Spanish flu' of 1918-1919, which is thought to have caused the deaths of 50 million people (more than the toll that resulted from the War itself). There is now increasing evidence that this 'plague' was the result of a strain of influenza similar to that of 'bird flu', outbreaks of which have generated much concern in recent years (Dobson 2015).

\section{Changes in human behaviour and the spread of disease}

The modern investigation of such pandemics reveals close links with rapid changes in human activities and lifestyles. All of the classic plagues are of infectious diseases, both bacterial and viral, whose spread was dramatically enhanced by increasing population densities, a process beginning with the change from a hunter-gatherer lifestyle more than 10,000 years ago, and by the increasing number of people travelling ever more rapidly over larger distances carrying the infectious agents; both of these factors increase the risk of transmission, particularly to populations that have not previously been exposed to such diseases. The changes in human behaviour that resulted in outbreaks of disease were, however, accompanied by an increasing realisation of their causes and of effective means of prevention and treatment. Improvements in sanitation and hygiene, and the development of vaccines and of antibiotics, have been dramatic, such that in much of the world the chances of dying from the classic infectious diseases that plagued humanity until modern times have been hugely reduced. The risk of pandemics has not been completely abolished, however, as resistant strains of many diseases that were thought to be have been largely suppressed, such as tuberculosis, have emerged, and 'new' types of disease have appeared, such as HIV/AIDS and Ebola (Dobson 2015; Peacock 2014). Even in such cases, however, modern science has so far managed to limit significantly the severity of these more recent 'plagues'.

The decline in the number of cases of infectious disease has led to greatly increased lifespans in most parts of the modern world; life expectancy in the most highly developed countries is now about 80 years compared to approximately 50 years a century ago. The result, however, has been a large increase in cases of non-infectious conditions, notably cancer and cardiovascular disease, which are now the greatest causes of death in much of the world outside sub-Saharan Africa. In the last few decades, however, there has been a dramatic increase in another group of disorders, collectively called dementias, of which the most common is Alzheimer's disease (AD) (Knowles et al. 2014). Dementia has recently been called a 'twentyfirst century plague', and indeed its rapidly increasing incidence and currently incurable nature is giving rise to the type of fears that in the past were associated with the plagues of infectious diseases of the type discussed above.

\section{The increasing number of cases of Alzheimer's disease}

Alzheimer first described the disease that bears his name in a lecture in 1906, after studying the case of a 51-year old woman, Auguste Deter who was admitted to the Frankfurt Asylum suffering from severe dementia. At the time, her case was a curiosity, and indeed it is evident that she was suffering from a rare early onset form of AD. Indeed it was only some 40 years ago that $\mathrm{AD}$ began to be recognised as an increasingly common condition among the elderly, but since then the number of cases has increased dramatically within our ageing populations (Alzheimer's Association 2015). The number of cases in the USA, for example, now exceeds 5 million, and it is estimated that there are some 40 million sufferers worldwide. This number is anticipated to rise to about 135 million by 2050 , of whom more than $70 \%$ will be in low or middle income nations; $\mathrm{AD}$ is, therefore, no longer a phenomenon confined to the wealthy nations of the world (Fig. 1). This increase in the number of cases is entirely attributable to the increasing number of people over the age of 65 , where the incidence of symptoms is between 1 and $2 \%$ of the population, rising to between one-third and one-half of those living to the age of 85 .

The financial burdens of this condition are enormous because of the need for continuous care of patients, often for years. In the USA, for example, the costs of AD are estimated to approach $\$ 200$ billion this year and are predicted to exceed $\$ 1$ trillion by 2050 (Fig. 2); indeed in the next 35 years, the cost of care and associated issues for $\mathrm{AD}$ sufferers in the USA alone is anticipated to be approximately \$20 trillion. Moreover, the extremely debilitating and uniquely distressing nature of dementia has huge implications for the stability of society and tremendous challenges for the health care systems of the world. It is for this reason that dementia has recently become recognised as an emerging international catastrophe, resulting in the first G8 Dementia Summit in London in 2013 where the need to increase spending on research into the nature of the disease, and into new approaches to the development of 


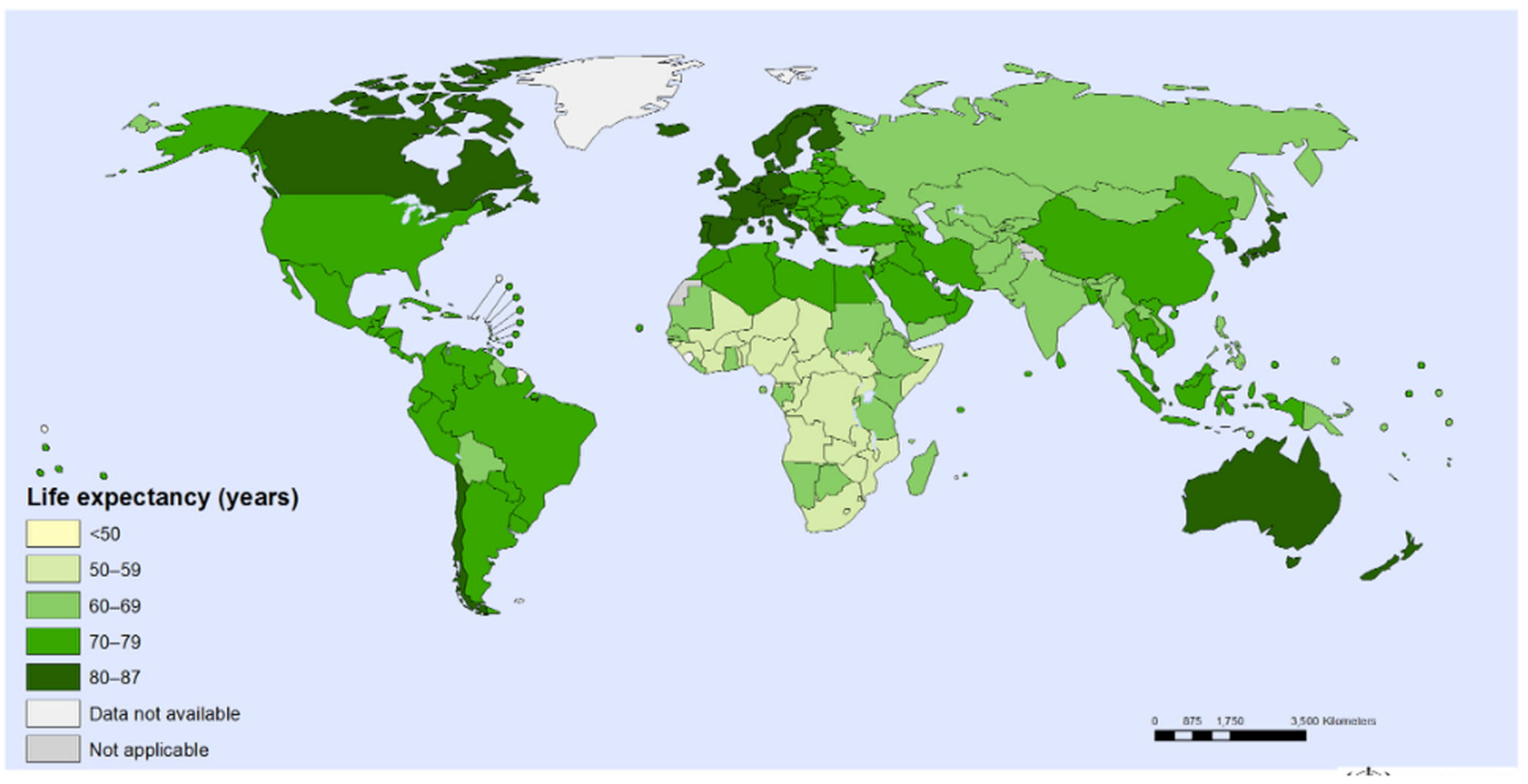

Fig. 1 Global life expectancy for both sexes in 2012. Source World Health Organization Map Production: Health Statistics and Information Systems (HIS), World Health Organization (2014)

Fig. 2 Predicted costs of Alzheimer's disease in the USA.

Taken from 'Changing the Trajectory of Alzheimer's Disease: A National Imperative'. Alzheimer's Association, May 2010. See also Alzheimer's Association (2015)

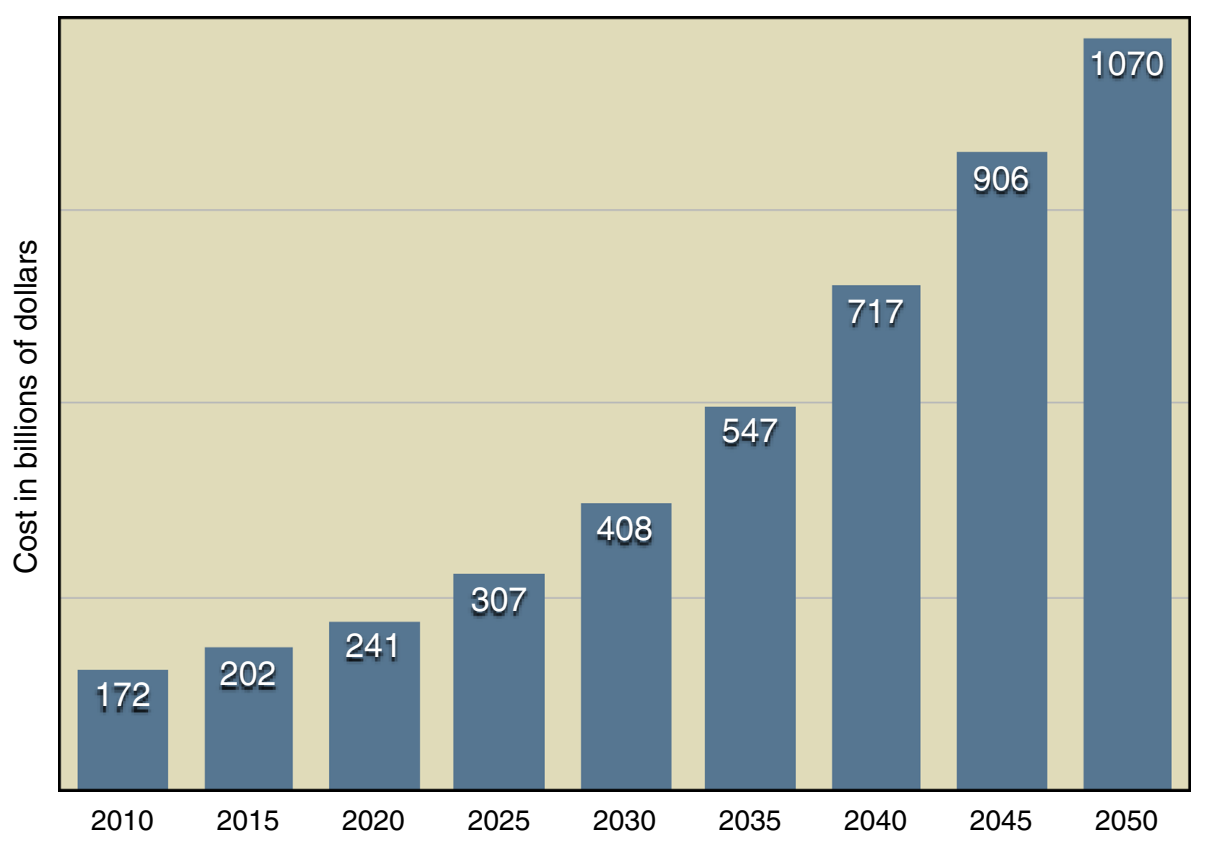

therapeutic strategies for its prevention and treatment, was clearly recognised.

\section{Alzheimer's disease and amyloid deposition}

It was evident from the earliest autopsies of patients who had died with $\mathrm{AD}$ that a characteristic feature of this condition is the presence of proteinaceous deposits within the brain. Indeed, these deposits were named 'amyloid' because they were found to stain with dyes that also stain for starch (the Latin word for which is 'amylum'). Indeed, it is now known that approximately 50 human diseases in addition to $\mathrm{AD}$ are associated with amyloid deposits (Chiti and Dobson 2006), including other neurodegenerative conditions such as Parkinson's disease (PD), Huntington's disease (HD) and motor neuron disease (MND), also known as amyotrophic lateral sclerosis (ALS). This group 
of diseases also includes systemic amyloidoses, where proteinaceous deposits are widespread in organs such as the liver, kidney and spleen; type II diabetes, where the deposits are found in the pancreas; and dialysis-related amyloidosis (DRA), where the deposits are formed in joints and skeletal tissue, and are a consequence of long-term hemodialysis procedures used to treat patients with kidney failure. In each of these conditions, the deposits are predominantly composed of one specific protein, which differs from one disease to another, and are often made up of masses of highly intractable thread-like fibrils, a few nanometers in diameter but often microns in length (Chiti and Dobson 2006).

Our interest in this class of diseases began in the mid1990s by complete chance, as we were focused at that time on the study of protein folding (Radford et al. 1992). One of the proteins that we were studying in greatest detail was lysozyme, an antibacterial enzyme, for which we had established many of the key determinants of the process through which it folds to its functional state. It then emerged that several familial mutations exist and that some give rise to a fatal form of systemic amyloidosis, bringing this enzyme into the group of proteins associated with protein deposition diseases (Booth et al. 1997). Further research on this system resulted in the discovery that these mutations greatly increased the probability of the protein misfolding, and that the misfolding process resulted in the conversion of the protein into pathogenic amyloid fibrils.

One of the fascinating questions that then arose concerned the nature of these fibrils and the determinants of their structure, which X-ray fibre diffraction studies had revealed as possessing a common 'cross- $\beta$ ' architecture consisting of $\beta$-strands oriented perpendicular to the fibril axis, which then form $\beta$-sheets oriented parallel with this axis (Sunde and Blake 1997). The existence of a common architecture for proteins in amyloid fibrils contrasts strongly with the fact that the native states of proteins are extremely varied, forming intricate and complex folds that are determined by the sequence of the amino acid building blocks in the polypeptide chains of which they are composed.

\section{The generic nature of the amyloid state}

Clues to the intrinsic nature of amyloid fibrils came from the unexpected, and initially chance, observation that in the laboratory it is possible to convert many proteins from their normal functional states into amyloid fibrils (Fig. 3) (Guijarro et al. 1998). Moreover, in a crucial series of experiments with polypeptide sequences in which all the amino acid residues are the same, such as polythreonine, it became evident that, unlike their functional forms, the

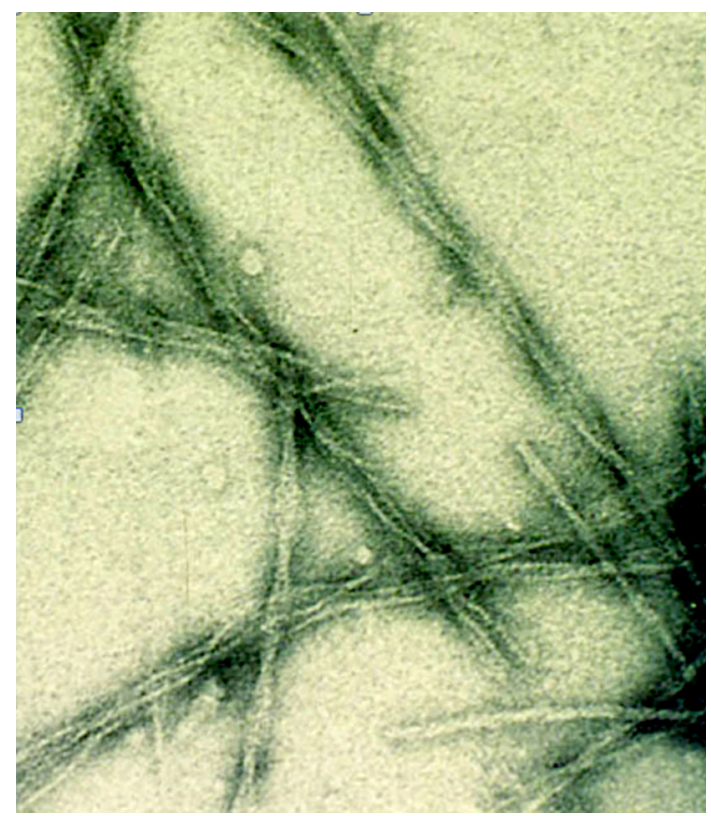

Fig. 3 Images of amyloid fibrils formed from the SH3 domain of PI3 kinase. The fibrils were formed in vitro by incubation of the protein (whose native structure is represented in Fig. 4) at low pH (Guijarro et al. 1998). Although the SH3 domain has no links with disease, the fibrils (that have diameters of just a few nanometers) have all the characteristics of the fibrillar aggregates isolated from the tissue of patients suffering from amyloid diseases such as Alzheimer's and Parkinson's disease (Chiti and Dobson 2006). This observation gave rise to the concept that the amyloid structure is a common or 'generic' form of protein architecture albeit, as we discuss below, it is one that is generally observed in nature only in pathological states (Dobson 1999)

architecture of the amyloid state of a protein is not encoded in its sequence (Fändrich and Dobson 2002). This observation has led to the realisation that there are two alternative highly organised forms of proteins, the unique functional native states and the generic amyloid state (Fig. 4) (Dobson 2008). The varied and complex structures of native proteins result from the dominance of specific interactions between the side-chains in the unique sequences of those proteins selected by evolution for functional purposes. By contrast, the structure of the amyloid state is dominated by the interactions of the polypeptide main chain that is common to all proteins; the amyloid structures of different proteins, therefore, adopt a common core structure, albeit with variations in the spacing of the $\beta$-sheets and other details, such as the way the various component protofilament structures assemble together to form the mature fibrils (Fändrich and Dobson 2002).

The atomic-level structure of an amyloid fibril, an 11-residue peptide from the protein transthyretin, has recently been determined by a combination of biophysical techniques, particularly solid-state NMR spectroscopy and cryo-EM (Fitzpatrick et al. 2013). The structure (Fig. 5) 

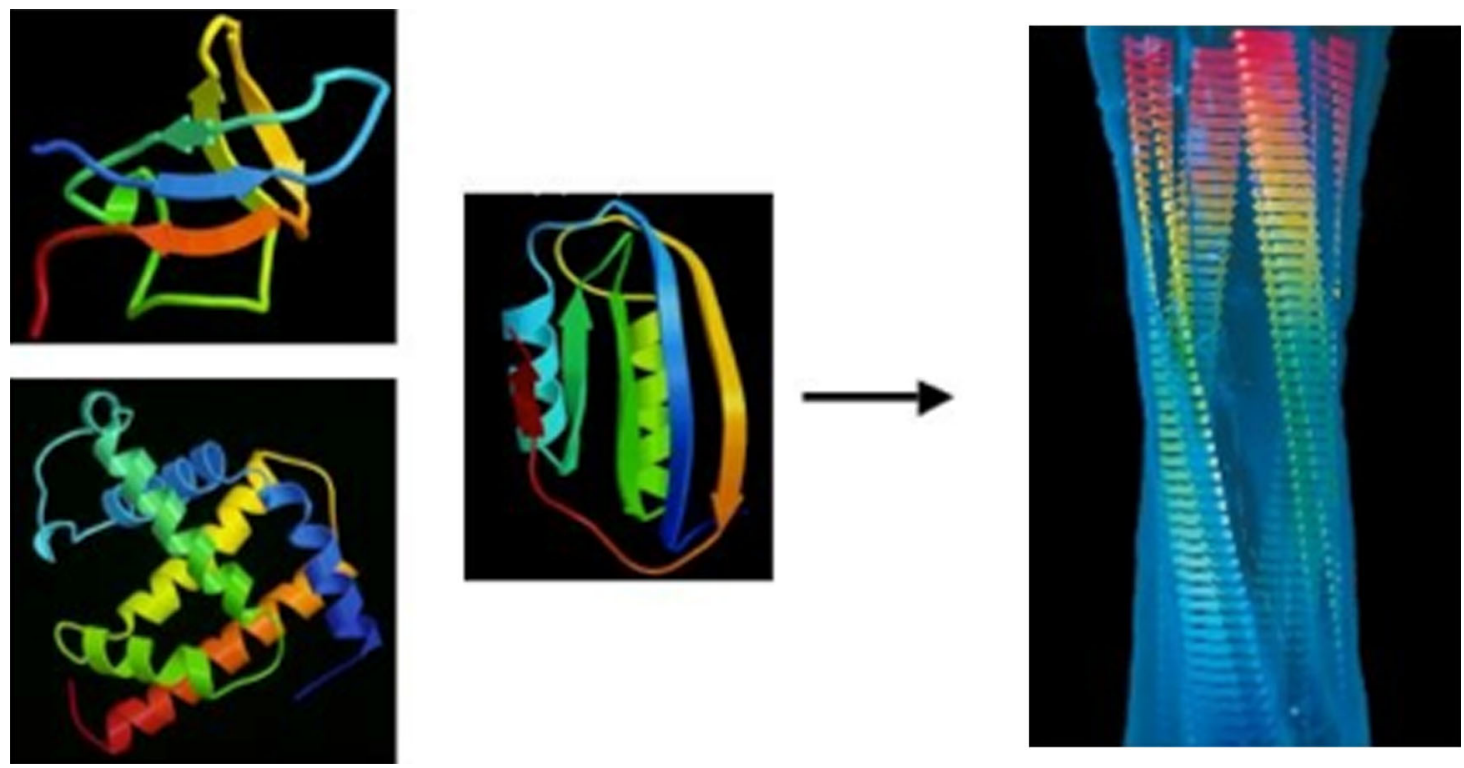

Fig. 4 Comparison of examples of native and amyloid structures of protein molecules. On the left are ribbon diagrams of the native structures of three small proteins: an SH3 domain (top), myoglobin (bottom) and acylphosphatase (middle). The native structures differ in their topologies and contents of $\alpha$-helices and $\beta$-sheets resulting from the dominance of side-chain interactions within their highly evolved sequences. On the right is a molecular model of an amyloid fibril [image kindly provided by Helen Saibil, Birkbeck College, London from work reported in Jiménez et al. (1999)]. The fibril was produced from the SH3 domain whose native structure is shown on the left, and consists of four 'protofilaments' that twist around one another to form a hollow tube with a diameter of approximately $6 \mathrm{~nm}$. The $\beta$-strands (flat arrows) are oriented perpendicular to the fibril axis and are linked together by hydrogen bonds involving main chain amide and carbonyl groups, many of which are intermolecular, to form a continuous structure in each protofilament. The protofilaments are held together by much weaker interactions involving primarily sidechain contacts. As the main chain is common to all polypeptides, the core protofilament structures of fibrils from different sequences have common features, differing only in detail as a result of differences in the non-dominant effects of side-chain packing. The black arrow indicates that when the native states of globular proteins are destabilised, they tend to convert into the generic amyloid structure, as described in the text. From Dobson (2008) shows that the core of the protofilaments is composed of a pair of nearly flat $\beta$-sheets, a feature proposed earlier on the basis of cryo-EM studies of the SH3 protein discussed above (Guijarro et al. 1998; Jiménez et al. 1999), and suggests the presence of water-filled cavities of the type observed in the X-ray structures of amyloid-like peptide microcrystals (Nelson et al. 2005). The fact that the cross- $\beta$ structure is a characteristic arrangement of polypeptide chains is supported by the results of computer simulations (Auer et al. 2008), as well as by theoretical analysis and by the results of investigations of the role of the arrays of hydrogen bonds that are features of this molecular architecture (Knowles et al. 2007). Although the specific protein sequence does not itself define this overall architecture, it does play a major role in defining the relative propensity of a given protein to convert from its normal functional state to the polymeric amyloid state, a feature that turns out to be crucial in enabling proteins in living systems to avoid rapid conversion into their amyloid states that in many cases may be thermodynamically the most stable state of a protein molecule (Baldwin et al. 2011).

\section{The pathogenic consequences of amyloid formation}

The conversion of soluble protein molecules into amyloid fibrils is accompanied by the loss of biological activity, as the latter is generated by the proximity of the functional groups of specific side-chains that are brought together in the unique fold of the native state. The conversion of a protein into the amyloid states is, however, associated with more than a loss of biological activity. Biological systems have evolved in such a way that the correctly folded structures of protein molecules, and indeed other biological molecules, can be packed together at high densities within cells and extracellular space (Minton 2000). The amyloid state of a protein 

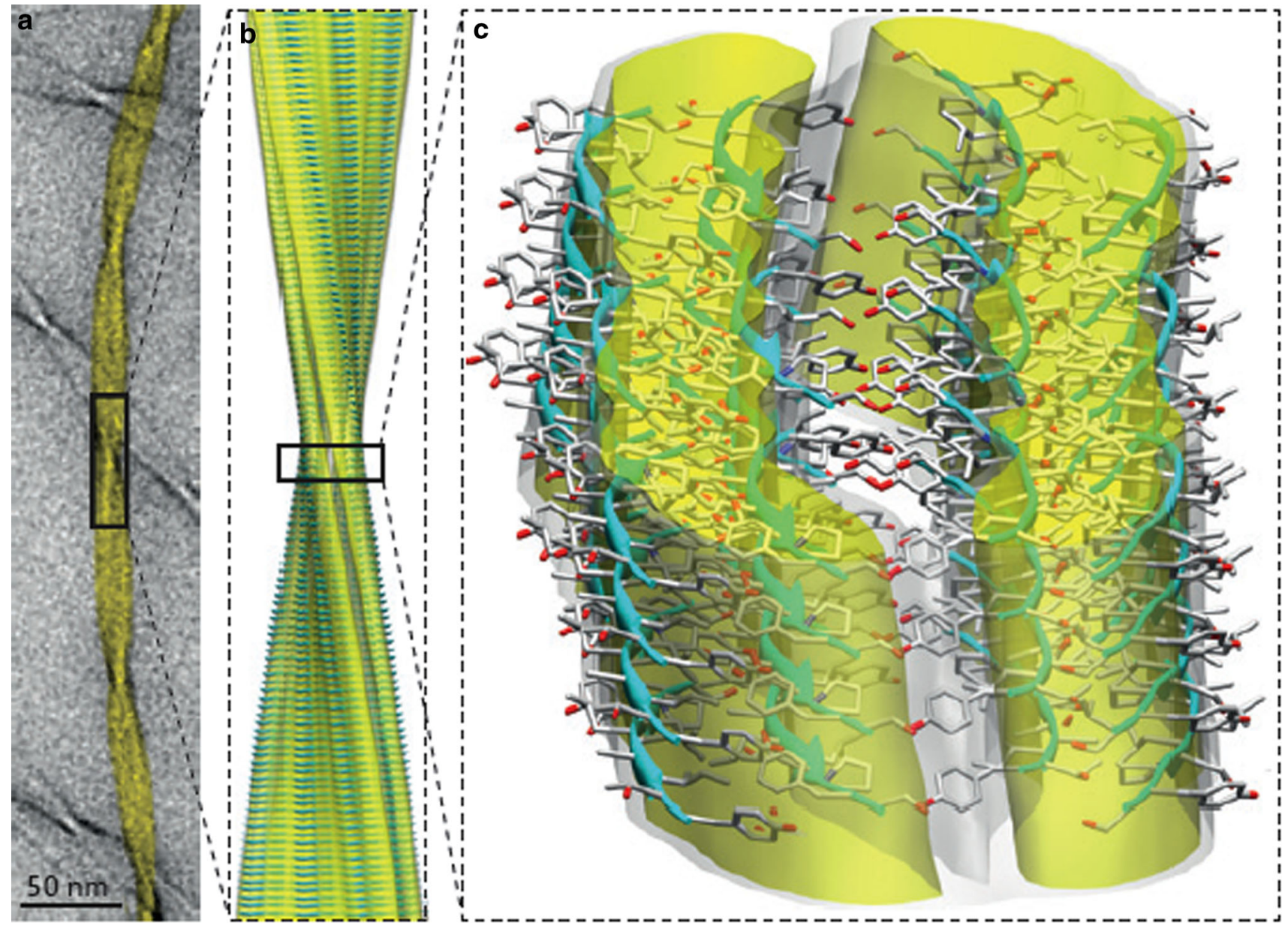

Fig. 5 Structure of an amyloid fibril at atomic resolution. The structure shown is one of several polymorphs of the amyloid fibrils that are formed from an 11-residue fragment of transthyretin (Fitzpatrick et al. 2013). The combination of cryo-electron microscopy imaging (a) with solid-state NMR analysis has enabled the determination of an atomic-level structure (b). A more detailed view (c) shows the hierarchical organisation of the amyloid fibril in which the three filaments that form the mature fibril illustrated here are in turn formed by pairs of cross- $\beta$ protofilaments, which are each composed of pairs of $\beta$-sheets. The fibril surfaces are shown as electron density maps, and the constituent $\beta$-sheets are shown in a ribbon representation; oxygen, carbon and nitrogen atoms are shown in red, grey and blue, respectively. From Knowles et al. (2014) molecule (except in the rare cases where this form of proteins has a functional role) is intrinsically misfolded, and so is inherently prone to interact inappropriately within the crowded and complex environment of a living system (Dobson 1999). The assembly into amyloid fibrils is a stepwise process that begins with the formation of small clusters of misfolded protein molecules that ultimately grow into mature fibrils. Moreover, these oligomeric species have been found generally to be much more toxic than the fibrils, a phenomenon that can be attributed to the greater exposure of 'sticky' hydrophobic side-chains that is associated with these pre-fibrillar species, which have much higher surface-to-volume ratios than do the larger and more structured fibrils (Bucciantini et al. 2002).
A key issue that arises in this context is why such species form and give rise to disease. Protein biosynthesis in living systems involves the generation of polypeptide chains having specific sequences of amino acids, the information for which is encoded in the genomic DNA, following which the folding process takes place in complex cellular environments. There is a finite possibility that any given chain will not fold correctly, but there are sophisticated and highly efficient systems within cells that can detect that a given polypeptide chain is misfolded and then target it for degradation; such a mechanism avoids the accumulation of potentially toxic aggregates, enables the component amino acids to be recycled and incorporated into new protein molecules, and in addition turns out to play a key role in the functioning of the immune system 
(Dobson 2003; Rubinsztein 2006). Indeed, both cells and extracellular spaces have a wide range of specialised proteins, many of which are called 'molecular chaperones' as they discourage inappropriate interactions during protein folding, whose purpose is the prevention of misfolding and its consequences (Hartl and Hayer-Hartl 2009). It is now clear that misfolding disorders such as Alzheimer's disease result at the most fundamental level from the failure of such protective mechanisms, with the result that pathogenic forms of proteins can accumulate and progressively damage or destroy those cells in their vicinity (Dobson 2003).

\section{Amyloid formation and the loss of protein homeostasis}

The actions of molecular chaperones and other systems that prevent misfolding play a key role in the maintenance of protein homeostasis, the mechanism by which every living system maintains the appropriate balance of the generation and degradation of proteins that enables all the normal functions of the organism to be carried out. Once such protective systems fail to prevent the progressive aggregation of one or more proteins, such homeostasis is lost and pathology results (Balch et al. 2008). It is clear that the ability to avoid protein aggregation is an important factor in biological evolution, and that all our proteins are able to remain in their functional states under normal physiological conditions. Nevertheless, recent evidence indicates that most proteins within their biological environments are at the highest concentrations at which they can remain soluble (Tartaglia et al. 2007). The reason for this situation is likely to result from the fact that the sequences and structures of protein molecules have evolved to enable them to be stable at the concentrations that are required for their optimal function. As random mutations will in general reduce their stability and solubility, proteins in living systems will tend to be just capable of remaining in their functional states under normal conditions.

This conclusion enables us, at least qualitatively, to be able to rationalise the reasons that protein misfolding diseases-virtually unknown a century ago-are now becoming frighteningly common (Dobson 2002; Knowles et al. 2014). Many of these 'modern diseases' can be attributed to the fact that we are now living to ages unprecedented in all of human history, and as we get older our protective mechanisms have an increasing tendency to fail, for example because proteins have an increasing tendency to be damaged by processes such as oxidative stress, and as the production of energy required for efficient protection starts to decline, resulting in a loss of protein homeostasis. Indeed, it appears that certain proteins are particularly vulnerable under such circumstances, many of which are in the pathways associated with neurodegenerative diseases, as they are 'supersaturated', that is they accumulate to levels that make them particularly vulnerable to aggregation (Cyriam et al. 2015). Other misfolding disorders are associated with different features of modern life, for example increasing obesity can change the levels of crucial protein hormones in the pancreas and result in type II diabetes. Another example is dialysis-related amyloidosis, as prolonged treatment by dialysis for kidney failure changes the levels of some serum proteins, notably $\beta 2$-microglobulin whose concentration can be increased by well over an order of magnitude, resulting in its slow deposition in joints and skeletal tissue.

In addition, the familial amyloid diseases, such as the systemic amyloidosis associated with lysozyme or the small fraction of cases of Alzheimer's disease with early ages of onset, can be attributed to the effects of mutations that reduce the solubility and stability of the proteins involved. A particularly dramatic example of these principles has come from the study of transgenic organisms such as fruit flies that express the 42-residue protein fragment $(\mathrm{A} \beta 42)$ that is the major component of the aggregates that form in Alzheimer's disease. By using the knowledge of the effects of amino acid substitutions on the propensity of a given protein to aggregate, it has been possible to design mutations within the sequence of $\mathrm{A} \beta 42$ that increase or decrease its propensity to aggregate and form amyloid deposits (Luheshi et al. 2007). Insertion of these mutational variants into the transgenic flies (Fig. 6) shows a remarkable correlation between aggregation propensity and neuronal damage (as revealed by locomotor ability and lifespan), a finding that both reveals the role of the aggregation process in neurodegeneration and the impact that the aggregation of a single (in this case non-essential) protein can have on the viability of a complex living system.

\section{Towards therapeutic intervention in amyloid diseases}

One of the key advances in our understanding of Alzheimer's disease and related conditions has been the realisation that for many proteins, particularly those that are relatively small or have been fragmented by proteolytic enzymes, the amyloid state can be more stable thermodynamically than the native state even under physiological conditions (Baldwin et al. 2011). The reason for the ability of such species to resist the conversion from their soluble forms into the amyloid state is, therefore, strongly dependent on the kinetics of this transition, the details of which are now beginning to emerge. It is now clear, for example, that the process of amyloid fibril formation involves a variety of distinct steps (Knowles et al. 2009). The first of these steps involves a primary nucleation event, in which 
Fig. 6 The effect of mutations in the sequence of the 42-residue human Alzheimer dysfunction in transgenic fruit flies (Luheshi et al. 2007). The upper left panel (a) illustrates a climbing assay of flies sequence (left) and two mutational variants predicted to reduce the peptide's aggregation propensity; the more mobile the flies the higher up the tube they can climb. The right hand upper panel (b) represents a similar experiment with flies expressing the $\mathrm{A} \beta$-peptide containing the E22G "Arctic mutation" (left hand tube). The two right hand tubes are of peptides that contain mutations that decrease the propensity to form prefibrillar aggregates (protofibrils) in particular. The lower panel (c) shows the degree of correlation between the relative locomotor activity of a series of mutational variants against their predicted propensities to form protofibrils. From Dobson (2008) A $\beta$-peptide on neuronal expressing the wild-type
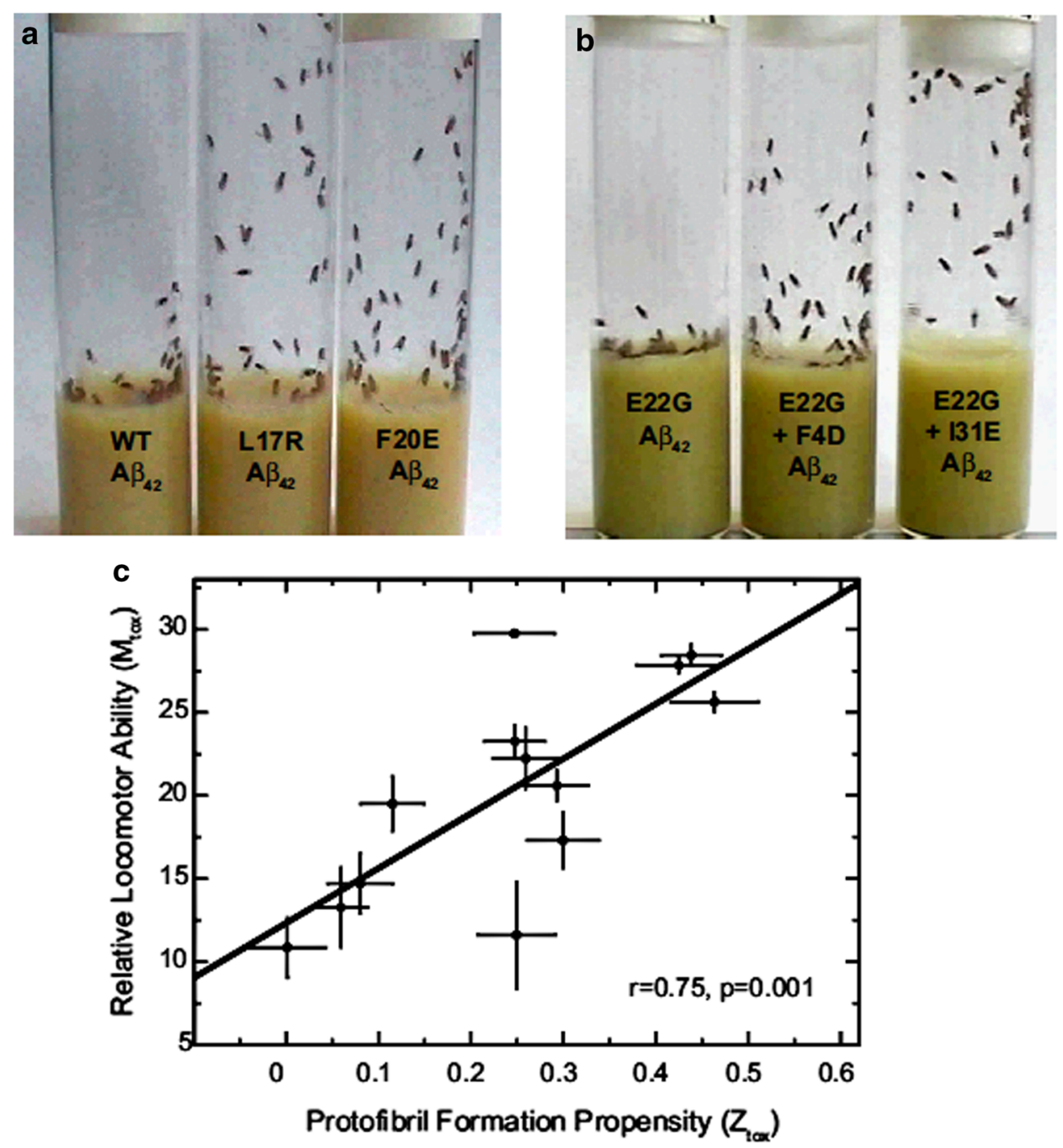

two or more soluble monomeric species interact with each other (Fig. 7). The resulting oligomers can then grow through sequestration of further soluble species, and in addition proliferation can result from secondary processes including fragmentation and surface-induced nucleation (Cohen et al. 2012). For filamentous aggregates, fragmentation increases the number of fibril ends from which growth can occur, and further nucleation can occur on the surfaces of the growing aggregates (Fig. 6). The aggregation reaction, therefore, includes a feedback loop such that the growth of aggregates catalyses the formation of more aggregates, leading to their rapidly increasing proliferation (Cohen et al. 2012, 2013).

The development of a multitude of experimental and theoretical techniques, ranging from single molecule measurements to mathematical modelling, has now made it possible to extract the rates of the different microscopic steps from experimental measurements of the kinetics of macroscopic amyloid formation under a series of defined conditions. In the case of the AD-related A $\beta 42$, analysis of experimental data has revealed the importance of the catalysis of the aggregation reaction by secondary nucleation on the surfaces of those aggregates that have already formed (Cohen et al. 2013). Moreover, studies of the effects of a variety of molecular chaperones reveal that different chaperones act on different microscopic steps in the aggregation process. Of particular interest is the fact that some chaperones target the initial primary nucleation process, but if such defenders fail to prevent the formation of aggregates, however, others are available as a secondstage defence mechanism. Indeed, it is clear, for example, that specific chaperones are able to switch off very selectively the catalytic process that drives the proliferation of the aggregation of $\mathrm{A} \beta 42$, and hence suppress the production of the most highly toxic oligomeric species associated with AD (Fig. 8) (Cohen et al. 2015). It is, therefore, evident that biological systems have developed a series of mechanisms by which prevention of the formation and 


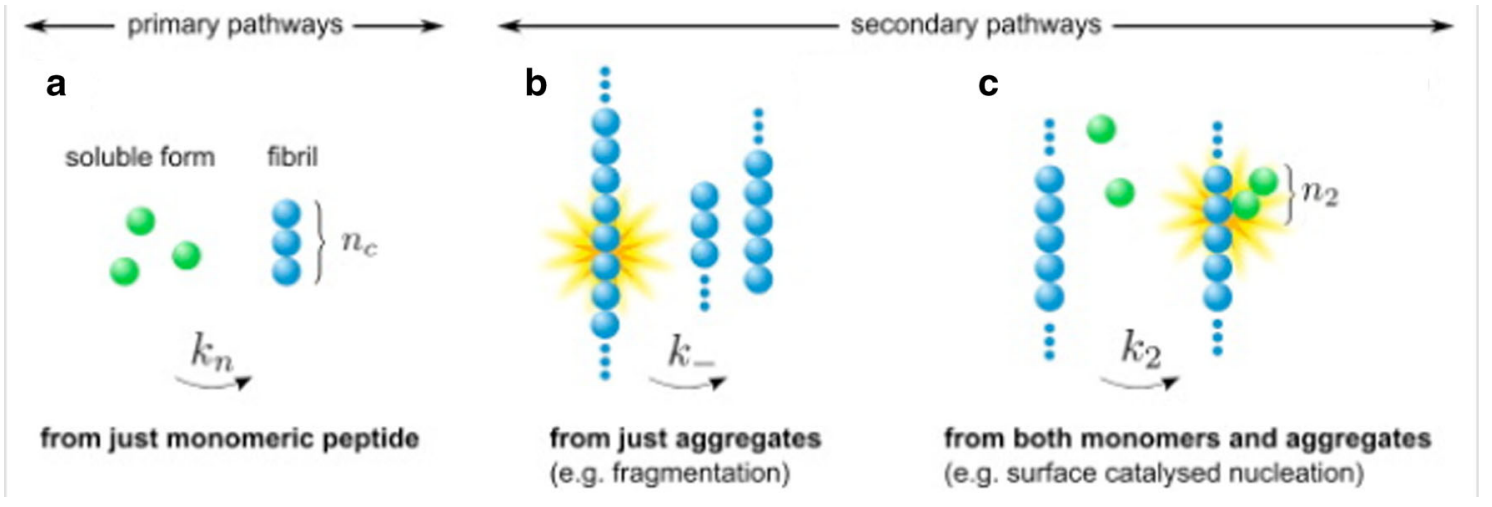

Fig. 7 A summary of the general classes of mechanism that create protein aggregates. a Primary nucleation pathways result in the formation of new aggregates from interactions solely between soluble monomers. Such aggregates can grow, and in the case of fibrillar species elongate, by the addition of further monomeric species. But the number of aggregates can increase by additional 'secondary' process including b monomer-independent events, such as fibril fragmentation, which generate new aggregates at a rate that depends

spreading of the species that are most damaging to cells can be achieved (Arioso et al. 2014).

The fact that biology has managed to generate such effective protection mechanisms, that result in few cases of $\mathrm{AD}$ developing until old age except where pathogenic mutations act to increase the risk of misfolding and aggregation, suggests that we might learn from the strategies that have evolved in living systems. The increasing emergence of misfolding diseases in the modern world, and indeed the experiments with fruit flies discussed above, show that relatively modest changes in the concentrations or properties of proteins are sufficient to cause a shift between health and disease. The corollary of this observation is that relatively modest changes in the other direction should be able to reduce the risk of these types of disorders, for example making it less likely that Alzheimer's disease will develop or progress until much more advanced ages (Arioso et al. 2014). One particularly attractive therapeutic strategy, therefore, would be to find means of enabling our natural defence systems to continue to act effectively in old age. We are therefore exploring ways of achieving this objective by searching for molecules that act, rather like the chaperones discussed above, as inhibitors of one or more of the microscopic processes that are involved in the aggregation process. Initial results suggest that small molecules that resemble existing types of pharmaceutical compounds may be able to act in this way (Arioso et al. 2014; Knowles et al. 2014), and we are now developing means of screening such potential drugs that are based on the techniques that we have developed to probe the intrinsic phenomena that underlie specific disorders. only on the level of aggregates already present in the solution, and c monomer-dependent events such as surface-catalysed secondary nucleation; this latter process creates new aggregates at a rate that depends on the concentrations of both monomeric protein and existing aggregates. The rate constants are labelled $k_{n}, k_{-}$and $k_{+}$, respectively, and the nucleation reaction orders with respect to the monomeric protein are denoted $n_{\mathrm{c}}$ and $n_{2}$. For further explanation see Cohen et al. (2012) from which this figure is adapted

\section{Looking to the future}

Research into the causes of misfolding disorders, notably Alzheimer's disease that is now reaching the level of a 'twenty-first century plague', has only been conducted in earnest for a few decades. By contrast, the bacterial and viral diseases that once plagued humankind were studied for centuries in the context of the progressive developments in our understanding of biological and medical science (Dobson 2015). Moreover, huge strides have also been made in the last 100 years or so in the development of the means of treating and preventing non-communicable diseases such as heart disease and cancer. Such diseases that were until recent times thought to be incurable are increasingly considered to be manageable and eminently treatable. Protective compounds such as statins reduce the risk of heart disease, and drugs such as tamoxifen have proved to be extremely effective in treating cancer. Although some advances in treatments over the ages have been fortuitous, the large majority has emerged through painstaking scientific study of the causes of the disease in question, followed by careful evaluation and development of therapeutic compounds that emerge from the testing and screening of possible lead compounds (Dobson 2013).

The progress that has been made in understanding the molecular origins of Alzheimer's disease is now at the point where rational development of therapies can now be explored (Knowles et al. 2014). The pace of advances in all aspects of science and medicine has never been greater, and collaborative efforts that bring together experts in different disciplines to address major problems have never been more strongly encouraged. The funding of research 


\section{Generation of fibrils}
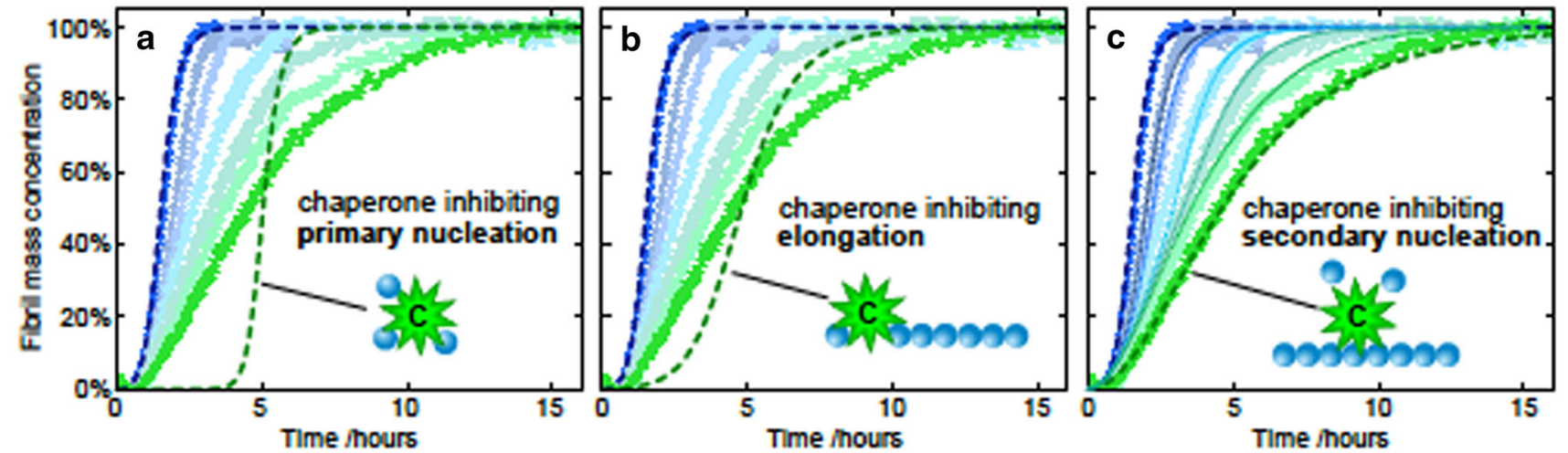

Generation of oligomers

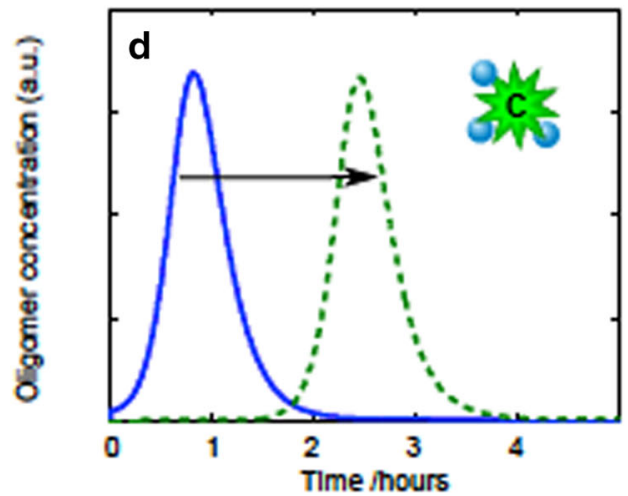

Fig. 8 Identification of the inhibition of secondary nucleation and evaluation of the consequences on the generation of toxic oligomers using kinetic analysis. a-c Kinetic reaction profiles for the aggregation of A 342 are shown from left (blue) to right (green) for reactions in the absence of the molecular chaperone called Brichos, and with 10, 20, 50, 75 and $400 \%$ A $\beta 42$ monomer equivalents of Brichos. The effect of Brichos saturates at a stoichiometry of approximately one monomer equivalent, and the blue dashed line is the integrated rate law for $\mathrm{A} \beta 42$ aggregation through primary and secondary nucleation using previously determined rate constants. The dashed green lines show predictions for the resulting reaction profiles when each of a primary nucleation, $\mathbf{b}$ fibril elongation and $\mathbf{c}$ secondary nucleation is inhibited by the chaperone. Note the characteristic differences in the change in the shape of the reaction profile in each case. The prediction for the case where the chaperone solely and entirely suppresses into dementia is at present only a fraction of that into cancer, and it is essential that this situation changes, and initiatives that replicate the US National Cancer Act of 1971, that led to a 'war on cancer', are needed to change this situation for neurodegenerative diseases. We need to understand yet more about the details of the underlying causes of these conditions and we need to develop new biomarkers that enable the progression of disease to be monitored rigorously and regularly; the latter are essential to enable clinical trials to be carried out effectively and rapidly.

With the progress that is being made in such directions we can therefore be extremely optimistic that misfolding diseases will follow infectious diseases and illnesses such as cancer into conditions that can increasingly successfully be prevented and treated (Dobson 2013; Knowles et al. 2014). In this manner dementia should be like the plagues of the past, in that its rapid rise in incidence will be followed by a steady decrease as it becomes a condition that can be managed effectively. Moreover, given the nature of misfolding diseases, there is little if any risk that methods will need to be found to prevent resistance to effective drugs, as is the case for infectious diseases and even some cancers, because the toxic agents of disorders such as Alzheimer's disease are not themselves evolving independently of the human hosts. There is every reason to believe, 
therefore, that these 'post-evolutionary' diseases, which have become prevalent in the modern world because of our success in addressing those diseases that have plagued humankind as a result of previous changes in lifestyle, will themselves become aspects of medical history rather than medical emergencies.

Acknowledgments This article is focused particularly on the studies carried out by members of my own laboratory and our many collaborators. There are too many people for me to mention them all in this section, but I would like to thank in particular, however, my close colleagues in Cambridge, Professors Michele Vendruscolo and Tuomas Knowles, who have contributed an incalculable amount to the work described in this article. In addition, I would like in the context of the Feltrinelli Award to express my huge appreciation of our long standing and uniquely valuable collaboration with members of the Department of Biochemistry at the University of Florence, particularly Professor Fabrizio Chiti, who was one of the most brilliant students with whom I have ever worked, and who has since then been closely involved in many of our most exciting projects in the field of amyloid formation for nearly 20 years, along with Professor Massimo Stefani and the late Professor Giampietro Ramponi, to both of whom I owe a huge debt as stimulating and inspirational collaborators. Finally, I would like to thank my wife, Mary Dobson, not just for her unfailing love and support, but for all that she has taught me about the nature and history of disease, and for the constant inspiration of her own work and writings on these and many other topics.

Open Access This article is distributed under the terms of the Creative Commons Attribution 4.0 International License (http://crea tivecommons.org/licenses/by/4.0/), which permits unrestricted use, distribution, and reproduction in any medium, provided you give appropriate credit to the original author(s) and the source, provide a link to the Creative Commons license, and indicate if changes were made.

\section{References}

Alzheimer's Association (2015) Alzheimer's disease: facts and figures

Arioso P, Vendruscolo M, Dobson CM, Knowles TP (2014) Chemical kinetics for drug discovery to combat protein aggregation diseases. Trends Pharmacol Sci 35:127-135

Auer S, Meersman F, Dobson CM, Vendruscolo M (2008) A generic mechanism of emergence of amyloid protofilaments from disordered oligomeric aggregates. PLoS Comput Biol 4:e1000222

Balch WE, Morimoto RI, Dillin A, Kelly JW (2008) Adapting proteostasis for disease intervention. Science 319:916-919

Baldwin AJ, Knowles TP, Tartaglia G, Fitzpatrick A, Devlin G, Shammas S, Waudby CA, Mossuto MF, Gras SL, Christodoulou J, Anthony-Cahill SJ, Barker PD, Vendruscolo M, Dobson CM (2011) Metastability of native proteins and the phenomenon of amyloid formation. J Am Chem Soc 133:14160-14163

Booth DR, Sunde M, Bellotti V, Robinson CV, Hutchinson WL, Fraser PE, Hawkins PN, Dobson CM, Radford SE, Blake CCF, Pepys MB (1997) Instability, unfolding and aggregation of human lysozyme variants underlying amyloid fibrillogenesis. Nature 385:787-793

Bucciantini M, Giannoni E, Chiti F, Baroni F, Formigli L, Zurdo J, Taddei N, Ramponi G, Dobson CM, Stefani M (2002) Inherent cytotoxicity of aggregates implies a common origin for protein misfolding diseases. Nature 416:507-511
Chiti F, Dobson CM (2006) Protein misfolding, functional amyloid, and human disease. Ann Rev Biochem 75:333-366

Cohen SI, Vendruscolo M, Dobson CM, Knowles TP (2012) From macroscopic measurements to microscopic mechanisms of protein aggregation. J Mol Biol 41:160-171

Cohen SI, Linse S, Luheshi LM, Hellstrand E, White DA, Rajah L, Otzen DE, Vendruscolo M, Dobson CM, Knowles TP (2013) Proliferation of amyloid- $\beta 42$ aggregates occurs through a secondary nucleation mechanism. Proc Natl Acad Sci USA 110:9758-9763

Cohen SI, Arosio P, Presto J, Kurudenkandy FR, Biverstål H, Dolfe L, Dunning C, Yang X, Frohm B, Vendruscolo M, Johansson J, Dobson CM, Fisahn A, Knowles TP, Linse S (2015) A molecular chaperone breaks the catalytic cycle that generates toxic $\mathrm{A} \beta$ oligomers. Nat Struct Mol Biol 22:207-213

Cyriam P, Kundra R, Morimoto RI, Dobson CM, Vendruscolo M (2015) Supersaturation is a major driving force for protein aggregation in neurodegenerative diseases. Trends Pharmacol Sci 36:72-77

Dobson CM (1999) Protein misfolding, evolution and disease. Trends Biochem Sci 24:329-332

Dobson CM (2002) Getting out of shape-protein misfolding diseases. Nature 418:729-730

Dobson CM (2003) Protein folding and misfolding. Nature 426:884-890

Dobson CM (2008) In: Zewail A (ed) Physical biology: from atoms to molecules. Imperial College Press, London, pp 267-279

Dobson M (2013) The story of medicine: from bloodletting to biotechnology. Quercus, London

Dobson M (2015) Murderous contagion: a human history of disease. Quercus, London

Fändrich M, Dobson CM (2002) The behaviour of polyamino acids reveals an inverse side chain effect in amyloid structure formation. EMBO J 21:5682-5690

Fitzpatrick AW, Debelouchina GT, Bayro MJ, Clare DK, Caparoni MA, Bajaj VS, Jaroniec CP, Wang L, Ladizhansky V, Muller SA, MacPhee CE, Waudby CA, Mott H, de Simone A, Knowles TPJ, Saibil HR, Vendruscolo M, Orlova E, Griffin RG, Dobson CM (2013) Atomic-resolution structure of a cross- $\beta$ amyloid fibril. Proc Natl Acad Sci USA 110:5468-5473

Guijarro JI, Sunde M, Jones JA, Campbell ID, Dobson CM (1998) Amyloid fibril formation by an SH3 domain. Proc Natl Acad Sci USA 95:4224-4228

Haines, Steckel RH (2014) A population history of North America. Cambridge University Press, Cambridge

Hartl FU, Hayer-Hartl M (2009) Converging concepts of protein folding in vitro and in vivo. Nat Struct Mol Biol 16:574-581

Jiménez JL, Guijarro JI, Orlova E, Zurdo J, Dobson CM, Sunde M, Saibil HR (1999) Cryo-electron microscopy structure of an SH3 amyloid fibril and model of the molecular packing. EMBO J 18:815-821

Knowles TPJ, Fitzpatrick AW, Meehan S, Mott HR, Vendruscolo M, Dobson CM, Welland ME (2007) Role of intermolecular forces in defining material properties of protein nanofibrils. Science 318:1900-1903

Knowles TPJ, Waudby CA, Devlin GL, Cohen SI, Aguzzi A, Vendruscolo M, Terentjev EM, Welland ME, Dobson CM (2009) An analytical solution to the kinetics of breakable filament assembly. Science 326:1533-1537

Knowles TPJ, Vendruscolo M, Dobson CM (2014) The amyloid state and its association with protein misfolding diseases. Nature Rev Mol Cell Biol 15:384-396

Luheshi LM, Tartaglia GG, Brorsson AC, Pawar AP, Watson IE, Chiti F, Vendruscolo M, Lomas DA, Dobson CM, Crowther DC (2007) Systematic in vivo analysis of the intrinsic determinants of amyloid beta pathogenicity. PLoS Biol 5:e290 
Minton AP (2000) Implications of macromolecular crowding for protein assembly. Curr Opin Struct Biol 10:34-39

Nelson R, Sawaya MR, Balbirnie M, Madsen AO, Riekel C, Grothe R, Eisenberg D (2005) Structure of the cross-beta spine of amyloid-like fibrils. Nature 455:773-778

Peacock S (2014) Health care: bring microbial sequencing to hospitals. Nature 509:557-559

Radford SE, Dobson CM, Evans PA (1992) The folding of hen lysozyme involves partially structured intermediates and multiple pathways. Nature 358:302-307
Rubinsztein DC (2006) The roles of intracellular protein-degradation pathways in neurodegeneration. Nature 443:780-786

Sunde M, Blake CCF (1997) The structure of amyloid fibrils by electron microscopy and X-ray diffraction. Adv Protein Chem 50:123-159

Tartaglia GG, Pechmann S, Dobson CM, Vendruscolo M (2007) Life on the edge: a link between gene expression levels and aggregation rates of human proteins. Trends Biochem Sci 32:204-206 